\title{
Wireless Sensor Network in Environment Monitoring
}

\author{
Tianyu Kang \\ College of Computer and information science College of Software, Southwest University, Chongqing, 400715, China \\ Email: isabellak21@email.swu.edu.cn
}

\begin{abstract}
With the advantages of low cost and low power consumption, wireless sensor network has attracted lots of interests and showed a great research and practical value in recent years. The applications of wireless sensor networks are concluded and reviewed systematically. And then problems in existing wireless sensor network are proposed. Finally, the future development of wireless sensor network is prospected.
\end{abstract}

Keywords: wireless sensor network, environment monitoring.

\section{INTRODUCTION}

With the rapid development of wireless communication technology, embedded operation technology and sensor technology, wireless sensor network(WSN) appeared[1]. Because of its low cost and power consumption, it is more and more frequently applied in environment monitoring.

Wireless sensor network is a type of selforganization network which consists of quantities of miniature sensor nodes located in monitoring area[2]. Figure 1 is a typical wireless sensor network model. It consists of four parts - sensor node, sink node, communication network $(\mathrm{CN})$ and monitoring center. Sensor nodes are supposed to be deployed in monitoring area randomly or regularly. They are responsible for monitoring the target object, and each node can collect the data of its coverage area and process them through the embedded computing module. Then the data are

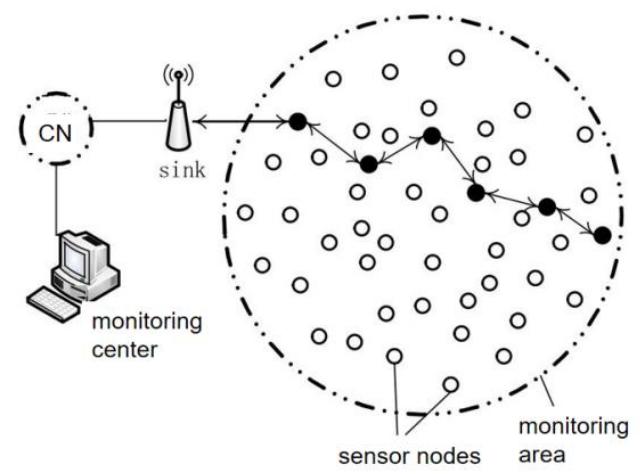

Figure 1 A typical structure of WSN system[3] transmitted to adjacent nodesor sink nodes in a multi-hop way. The sink node is a type of special node which is similar to the gateway. Its strong processing capacity, storage capacity and communication capacity enable it to bridge the wireless sensor network to the communication network so that it is convenient for the terminal to carry out real time operations through control center[1].

Modern WSN is more likely a novel type of distributed control system, which composed of a large number of sensor nodes distributed in the monitoring area. Compared with traditional sensor systems, the networking mode of WSN does not depend on fixed network facilities. Instead, sensor nodes form a selforganizing network according to certain transmission protocols and relay information through multi-hop routes. The nodes can communicate with remote nodes through intermediate nodes. And they can join and exit the network at any time without affecting other nodes. So the WSN has the advantages of flexible layout, strong invulnerability, wide distribution range and so on. Because of the above advantages, wireless sensor networks have significant research value and great practical value in military, industrial, agricultural, environment monitoring, medical, smart home, space and ocean exploration and other fields. It has attracted great attention from military departments, industry world and academia in many countries[4-5].

Environment monitoring is a typical application of wireless sensor networks. As human are more and more concerned about the environment, the need of technologies for environment monitoring is bigger and bigger. In traditional way, it is very difficult to collect the original data. For example, the data collection and 
procession of traditional atmospheric environment monitoring depends on manual sampling[6]. Monitors collect samples on the spot for a period of time, and then take them back to the laboratory for analysis. Therefore, only the average data of the gas in a specific period of time can be obtained but not the real-time data and the results are greatly affected by human. In addition, the health of monitors can be damaged if there are some poisoned gases on the spot. WSN can perfectly deal with these problems. The network has the ability of communication and the large number of nodes can collected more data as well as reduce the impact of human. Because of these advantages, the environment monitoring system based on WSN advances the traditional environment monitoring system and has a bright prospect. Therefore, the research on the application of WSN in the environment monitoring such as real-time collection, monitoring, processing, analysis and prediction, which can improve the efficiency and security of environment monitoring, is of great significance.

\section{HOT ISSUES AND KEY TECHNOLOGIES OF RESEARCH OF WIRELESS SENSOR NETWORK}

According to its function, structure of wireless sensor networks can be abstracted into five layers, namely, basis layer, network layer, middleware layer, data management and processing layer application development environment layer and application layer. Table 3 shows the structure of the wireless sensor network. There are many work and problems in each layer. The basic composition of each layer, hot issues and some key technologies of the wireless sensor network research are discussed as follow.

Table 1 Abstract structure of wireless sensor system

\begin{tabular}{|c|}
\hline Application layer \\
\hline Application development environment layer \\
\hline Data management and processing layer \\
\hline Network layer \\
\hline Basis layer \\
\hline
\end{tabular}

\subsection{Basis layer}

The basis layer takes the sensor set as the core, including the software and hardware resources of each sensor. The functions of this layer mainly include monitoring and information collection of objects and preliminary procession and transmission of collected information. The key technology of basic layer research is the software and hardware technology of wireless sensor network.

\subsection{Network layer}

The network layer takes communication network as the core, including communication network, various protocols supporting network communication and software and hardware resources. The functions of this layer include realizing the communication between sensors and sensors, sensors and observers and supporting multi-sensor cooperation to complete large tasks. The research issues of network layer mainly include protocols of network communication and various technologies of wireless sensor network.

\subsection{Data management and processing layer}

Data management and processing layer takes data management and processing software of sensor as the core. The main functions of this layer include data management, analysis and processing software system, which supports the storage, query, analysis and mining of collected data and provides effective support for user's decision-making. The research issues of this layer include data management, query, analysis and mining technology and data management system.

\subsection{Application development environment layer}

The application development environment layer provides an effective software development environment and tool for users to develop wireless sensor network system based on the basis layer, network layer and data management and processing layer. The research issues of this layer mainly include wireless sensor network programming language, applicationoriented network, sensor network data analysis, decision-making theory.

\subsection{Application layer}

The application layer is composed of software system for sensor network applications. The research issues of this layer mainly include the development of wireless sensor network application system.

\section{APPLICATIONS OF WSNS IN ENVIRONMENT MONITORING}

\subsection{Atmospheric environment monitoring}

Atmospheric environment monitoring system based on WSN is one of specific applications of WSN in environment monitoring. The existing monitoring system of gas composition has many shortcomings. For example, the system is too huge and difficult to install 
and the terminals heavily rely on wire communication. With the help of WSN, the atmospheric condition in a wide range can be monitored at real time, the composition of gases can be detected by different sensor probes and the leakage of toxic gases can be monitored timely and effectively according to the wind direction, wind speed, gas concentration and other parameters collected by the nodes. J Geng, X Zhou and B Zhang[6] proposed a network construction scheme of atmospheric environment detection system based on WSN by designing the hardware structure of wireless sensor network nodes for atmospheric environment monitoring. Z Wu et al.[7] developed a wireless sensor network system for monitoring harmful gases in living area, which can keep working for 3 weeks. The nodes in network transmit data directly to the sink node. The maximum data transmission rate of the node is $165 \mathrm{~m}$ $\mathrm{kb} / \mathrm{s}$, the effective transmission distance between buildings is $75 \mathrm{~m}$ and the transmission distance in the open area is $320 \mathrm{~m}$. Y Li, G Ji and J Han[8] designed the structure of multi-greenhouse environment monitoring system based on WSN by using ZigBee technology, Mesh mesh topology and $2.4 \mathrm{GHz}$ RF transceiver wireless module. And gave both software and hardware scheme for single-greenhouse environment monitoring. The system is stable and easy to expand, and the structure of it is simple. It realizes the flexible arrangement of sensors and improves the real-time monitoring ability of various parameters in the greenhouse environment.

\subsection{Water resources monitoring}

Wireless sensor network technology has unique advantages in water resources monitoring. Nodes in the network can measure various signals in the surrounding environment such as heat, infrared light, visible light and toxic substances. It is easy to deploy and does not need support of infrastructures such as cables. In addition, the sensor node is cheap and can be densely deployed in a large area of water, so it is convenient to analyse and use the spatial correlation of the collected information to obtain more accurate environment information[9-11]. L Zhao and J Fan[12] apply WSN combined with soft sensor and intelligent information processing technology, monitor the quality of wetland water environment remotely at real time and improved the detection accuracy of the remote real-time monitoring wireless sensor network system. W Bi and $\mathrm{H}$ Guo[13] uses the ZigBee protocol to form a wireless sensor network, which is used to monitor the marine water environment. The network system combines wireless sensing technology, embedded computing technology, modern network technology, wireless communication technology and distributed intelligent information processing technology, which greatly improves the sensors' ability to monitor various parameters of the ocean. The system adopts medium- and short-distance low-power wireless network, it has low radio frequency transmission cost and can adopt a variety of power supply modes according to circumstance.

\subsection{Geology monitoring}

Wireless sensor networks are also widely used in geology monitoring. The University of Southampton in the UK has launched the GlacsWeb system project[14], which aims to monitor and study glacier activities using wireless sensor networks. The system is deployed in the Briksdalsbreen Glacier in the Jostedalsbreen ice sheet in Norway. Sensors are deployed on, in and below the surface of the glacier to collect data and send them to the base stations on the glacier. The data collected by this project make a big contribution to the study of glacier movement and global warming. L Luo and Y Zhang[15] designed a new type of wireless sensor network for monitoring glaciers in extreme environment and gave the network structure and the transmission path of the data collected by sensor nodes. They came up with the problems in WSNs which need paying attention to such as the functions of operating system, sensor materials, energy consumption and communication capability of wireless sensor networks under the glacier. They also gave the measures of constructing all-weather observation network and realtime data display platform by using Google Earth, Google SketchUp and other technologies.

\subsection{Mine environment monitoring}

Accidents under coal mine often occur. The safety of underground workers is a big problem. Therefore, underground safety monitoring technology is very important. With the rapid development of coal production, the structure of mine has become increasingly complex, which makes the wired transmission mode of safety monitoring less convenient than the wireless transmission mode[16-18]. Wireless sensor network technology largely solves this problem, among which ZigBee technology is widely used. ZigBee technology is a two-way wireless network technology with short distance, low complexity, low power consumption, low data transmission rate and low cost[19-20]. Applying the ZigBee technology to sensors and establishing sensor networks is of great significance to effectively solve the problem of efficient and safe production under mines. Y Zhang, G Yuan and L Chen[21] designed a wireless positioning system based on ZigBee technology to solve the problem of underground communication and positioning.

\subsection{Military environment monitoring}

Wireless sensor network is composed of intensive, low-cost, and randomly distributed nodes. The self- 
organization and fault-tolerant ability make it avoid the collapse of the system due to the damage of some nodes in malicious attacks. This advantage makes the wireless sensor system largely advances the traditional sensor technology and makes it very suitable for harsh battlefield, It can play an important role in $\mathrm{C}^{4}$ ISRT system(command, control, communication, computing, intelligence, surveillance, reconnaissance and targeting), such as monitoring the equipment and materials of our army and conflicts, reconnoitring the terrain and defense system of enemy, positioning attack targets, assessing losses, reconnoitring and detect nuclear, biological and chemical attacks.

The US Army has launched a series of research projects in recent years to explore the application of wireless sensor networks in future wars. The Sens IT (Sensor Information Technology ) project funded by DAPAR enables soldiers to obtain battlefield information quickly and comprehensively by deploying wireless sensor networks composed of different types of sensors in the battlefield. The network has the characteristics of self-organization and selfconfiguration. It can dynamically adapt to the failure and aging of equipment, manage the movement of sensor nodes and respond to tasks and network requirements[22]. Ohio is developing A Linein the Sand, a wireless sensor network system. This system can scatter electronic trip wires to any place to detect targets. This capability means a special military use, such as reconnaissance and positioning of enemy tanks and other vehicles. Wireless sensor network technology foreshadows bringing new electronic eyes and ears to the battlefield, which 'can change the battlefield environment in the next few decades'[22].

\subsection{Others}

Wireless sensor network is of great important in many other circumstances such as mine environment monitoring, military environment monitoring, agriculture and animal husbandry environment monitoring, forest environment monitoring, Pipeline transportation monitoring and so on[4].

\section{FUTURE}

At present, although the industry of wireless sensor networks is booming, there are many problems. There are many factors that restrict the development of wireless sensor networks, including power consumption of nodes, transmission network, working environment, topology, cost, fault tolerance and so on. Taking power consumption of nodes for an example, as most of the power supplies of nodes are built-in batteries, how to efficiently use the limited energy to play a more lasting role has become an important issue. In the topology of data node networks, those sensor nodes that are inaccessible and unattended by human tend to frequently breaking down, which makes the topology network maintenance of wireless sensor networks a big problem.

These problems can not be solved in isolation. Only when we consider them together and find the best balance among them, can we make the wireless sensor network play the most effective role. With the improvement of wireless sensor network technology, wireless sensor networks will gradually become a essential part of people's daily life and will be everywhere in the near future.

\section{CONCLUSION}

Nowadays, because of the rapid pace of exploration of internet of things and intelligentialize, the need of wireless sensor network, the wireless network with low cost and low power consumption, is growing. Many researchers have great interests in the applications of wireless sensor network in environment monitoring. Lots of researches such as the scheme for a network construction scheme of atmospheric environment detection system based on WSN[6], the wireless sensor network used for marine water monitoring[13], the GlacsWeb system project[14] and so on had been conducted. It turns out that there is big benefits and a bright, promised prospect of applying wireless sensor network in environment monitoring. Meanwhile, there are many issues such as power consumption of nodes, transmission network, working environment, topology, cost, fault tolerance and so on need to be taken into consideration when developing the wireless sensor network. But these issues will be no more worries one day with the efforts of researchers.

\section{REFERENCES}

[1] I.F. Akyildiz, W. Su, Y. Sankarasubramaniam, et al. Wireless sensor network: A survey[J]. Computer Networks, 2002, 38(4): 393-422.

[2] W. Wang, T. Wang, Q. Wu, et al. Survey of delayconstrained data collection with mobile elements in WSN[J]. Journal of Computer Research and Development, 2016, 53(11): 1-19.

[3] H. Chen. Overview of Wireless Sensor Network[J]. Software, 2016, 37(09): 105-108.

[4] J. Jia. Coverage Control and Node Deployment Technologies in Wireless Sensor Networks[M]. Shenyang: Publisher of Dongbei University, 2013.

[5] T. Arampatzis, J. Lygeros, S. Manesis. A Survey of Applications of Wireless Sensors and Wireless Sensor Networks. In: Proceedings of the 2005 IEEE International Symposium on Mediterrean 
Conference on Control and Automation Intelligent Control, 2015, pp. 719-724.

[6] J. Geng, X. Zhou, B. Zhang. An atmosphere environment monitor system based on wireless sensor network[J]. Journal of Xi-hua University: Natural Science, 2007, 26(4): 44-46, 96.

[7] Z. Wu, Z. Liu, J. Liu, et al. Wireless sensor networks for living environment monitoring. In: Proceedings of the 2009 WRIWorld Congress on Software Engineering, Xiamen, 2009, pp. 22-25.

[8] Y. Li, G. Ji, J. Han. Application of the wireless sensor network in environment monitoring system of greenhouse[J]. Process Automation Instrumentation, 2010, 31(10): 61-64.

[9] F. Hong, H. Chu, Z. Jin, et al. Review of recent progress on wireless sensor network application[J]. Journal of Computer Re-search and Development, 2010, 47 (Supp.2): 81-87.

[10] Z. Guo, H. Luo, F. Hong, et al. Current progress and research issues in underwater sensor networks[J]. Journal of Computer Research and Development, 2010, 47(3): 377-389.

[11] P. Gong. Progress in recent environmental applications of wireless sensor networks[J]. Journal of Remote Sensing, 2010, 14(2): 387-395.

[12] L. Zhao, J. Fan. Research on key technology of water resources monitoring system based on wireless sensor networks[J]. Computerand Modernization, 2010(8): 85-88.

[13] W. Bi, H. Guo. Design of ocean water environment monitoring systembased on wireless sensing network[J]. Electronic Technology, 2010(8): 68-71.

[14] K. Martinez, J.K. Hart, R. Ong. Deploying a wireless sensor network in Iceland. In: N. Trigoni, A. Markham, S. Nawaz. Lecture Notes in Computer Sciences: Proceeding of the 3rd International Conference on GeoSensor Networks, Berlin, Springer, 2009, pp. 131-137.

[15] L. Luo, Y. Zhang. Application research of wireless sensor networks in glacial environment monitoring $[\mathrm{J}]$. Journal of Glaciology andGeocryology, 2008, 30(6): 1018-1023.

[16] Witrante, D. Innocenzo, A. Sandoug, et al. Wireless ventilation control for large-scale systems: The mining industrial case[J]. International Journal of Robust and Nonlinear Control, 2010, 20(2): 226251.

[17] X. Jing, W. Wang, L. Hei, et al. Application of wireless sensor network in coal mine safety intelligent monitoring system $[\mathrm{J}]$. Coal Technology, 2009, 28(4): 93-97.

[18] Y. Kang, Y. Hu, C. Xie, et al. Application of WIFI in mine emergency WSN[J]. Journal of Henan Polytechnic Univer-sity: Natural Science, 2010, 29(5): 635-638.

[19] D. Gislason, T. Gillman. ZigBee wireless sensor networks[J]. Dr Dobb' s Journal, 2004, 29(11): $38-40$.

[20] F. Kiyoshi, T. Akira, Shigeruf. ZigBee technology for low-cost and low-power radio communication systems[J]. Journal of the Institute ofElectronics, Information and Communication Engineers, 2005, 88(1): 40-45.

[21] Y. Zhang, G. Yuan, L. Chen, et al. Mine wireless localization system based on ZigBee technology[J]. Zhongzhou Coal, 2010(6): 30-32.

[22] H. Wang, J. Elson, L. Girod, et al. Target classification and localization in habitat monitoring[J]. In: Proceedings of IEEE International Conference on Acoustics, Speech, and Signal Processing (ICASSY2003), Hong Kong, China, 2003. 